\title{
The effect of compression on solid-state properties of desloratadine and multicomponent crystal
}

\author{
Ahmad AINUROFIQ 1, 2*, Rachmat MAULUDIN ${ }^{1}$, Diky MUDHAKIR ${ }^{1}$, Sundani Nurono SOEWANDHI ${ }^{1}$ \\ 1 School of Pharmacy, Institut Teknologi Bandung, Ganesha 10, Bandung, 40132, Indonesia \\ 2 Department of Pharmacy, Sebelas Maret University, Ir. Sutami 36A, Surakarta, 57126, Indonesia \\ * Corresponding author. E-mail address: rofiq@mipa.uns.ac.id (A.A.); Tel. +62271663375; ORCID No: 0000-0003-2880- \\ 5321.
}

Received: 29 December 2017 / Revised: 05 March 2018 / Accepted: 11 March 2018

\begin{abstract}
Tablet pharmaceuticals manufacturing process involves several processes i.e. granulation, drying, milling, and compression. This study was conducted to determine the effect of compression of desloratadine (DES) and multicomponent crystal (MCC), which is a salt from DES, on their solid-state properties. A compression method for tablet formation was conducted using a hydraulic pressure machine with punch diameter of $13 \mathrm{~mm}$. Both DES and MCC were made into tablets with the same tensile strength, which is $1.36 \mathrm{MPa}$. DES and MCC powder and tablet were each evaluated for their physicochemical properties, including analysis of powder X-ray diffraction (PXRD), differential scanning calorimetry (DSC), Fourier transform infrared spectroscopy (FTIR), scanning electron microscopy (SEM), and tablet dissolution. PXRD diffractogram analysis results on DES and MCC showed that the degree of crystallinity decreased after the compression process. There were no polymorphic transformation in DES and MCC after compression. DSC analysis showed a decrease of enthalpy after DES and MCC are compressed. FTIR showed the same spectra pattern for DES and MCC powder and tablet. The SEM scans showed a tendency for interparticle attachment in DES tablets and none in MCC tablets. Meanwhile, dissolution, as indicated by dissolution efficiency, is only slightly decreased due to the compression process. DES and MCC analysis, all characteristics indicated changes of physicochemical properties after compression pressure, but those changes did not affect their dissolution results.
\end{abstract}

KEYWORDS: Desloratadine; multicomponent crystal; compression; solid-state properties; dissolution.

\section{INTRODUCTION}

The most common pharmaceutical products are in the dosage form of tablets. The production of tablets includes the process of mixing, drying, milling, extrusion, fluidization, granulation, compression, and coating. Compression process often leads to change the properties of active pharmaceutical ingredients, such as particle size, specific surface area, crystallinity, polymorph or crystal habit [1-2]. The effect of compression pressure on crystals during the manufacturing process can cause changes and disorder in the crystal, which affect deformation variation and fracture of solid powder physical properties [3-5]. Meanwhile, parameters such as morphology, melting point, solubility, and stability are dependent on the formation of crystals from drugs [6].

Desloratadine (DES) is a histamine $\mathrm{H}_{1}$ selective antagonist receptor that is widely used for anti-allergy and anti-inflammatory as a treatment option because it has no effect on the central nervous system, cardiovascular disorders [7-9]. DES is problematic physicomechanically with poor tabletability property, which is resolved by the formation of multicomponent crystal (MCC) in the form of salt [10-12]. The Structures of DES and MCC are shown in Figure 1a,b, respectively. DES is a weak base with pKa value of 8.65 [13]. DES is made into a MCC with benzoic acid (BA) in an equimolar ratio, using the solvent evaporation method dissolved in methanol [14]. The formation of MCC can be used to change the physicochemical properties of drugs by rearrangement of molecules to form a new crystal structure and control the molecules arrangement in the crystal lattice [10].

Variations in solid-state properties of active pharmaceutical ingredients (API), including DES and MCC will have an impact on manufacturing success from drugs formulation and therapy [15]. Crystallinity in several drugs is highly related to compression pressure, while tablet dissolution property is reported to be

How to cite this article: Ainurofiq A, Mauludin R, Mudhakir D, Soewandhi SN. The effect of compression on solid-state properties of desloratadine and multicomponent crystal. Marmara Pharm J. 2018; 22 (4): 570-577. 
affected by the degree of crystallinity. Therefore, a consideration is needed to understand the response of solids to mechanical treatment during production.

There are several results of research on mechanical studies in the tableting process, but there is little research on the physicochemical changes caused by compression pressure due to tableting [16]. There are no reports that revealed the effect of compression on the characteristics of the solid-state of DES and its MCC salt form. MCC as a result of new crystal finding is very necessary to be performed a pre-formulation study. This study was aimed at determining the effect of compression pressure on physicochemical properties, i.e., interparticle attachment, degree of crystallinity, and dissolution of DES and MCC. It is important to investigate the effect of compression pressure due to tableting on physicochemical properties and dissolution effects, which may cause changes in dissolution due to the pharmaceutical preparation process.

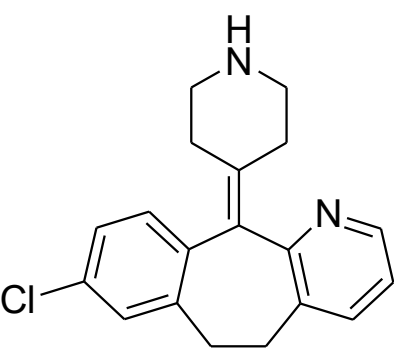

(a)

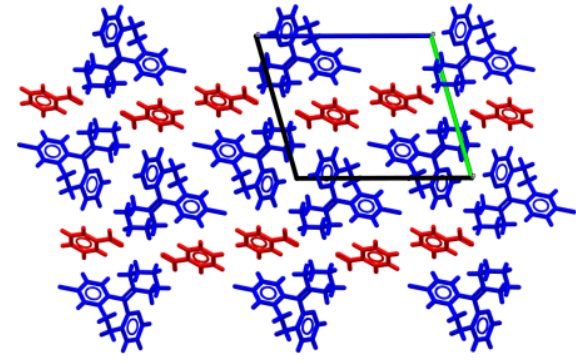

(b)

Figure 1. Chemical structure of (a) desloratadine and (b) multicomponent crystal of desloratadine.

\section{RESULTS AND DISCUSSION}

\subsection{Physicochemical Properties Evaluation}

\subsubsection{Powder X-ray diffraction (PXRD)}

PXRD is a useful method to determine the existence of polymorphism, amorphous phase, crystallinity and modification of crystal habit in solid drugs. Diffractogram from DES and MCC before (powder) and after (tablet) compression process are presented in Figure 2. Compression treatment was observed at $2 \theta$ angles between $5^{\circ}$ to $45^{\circ}$ did not change the peak position of DES and MCC, which indicated that there was no polymorphic transformation that occurred in DES and MCC after compression process. This was shown by changes in crystallinity as listed in Table 1 . The degree of crystallinity of DES decreased by $9.3 \%$, while MCC decreased by $6.4 \%$ after compression.

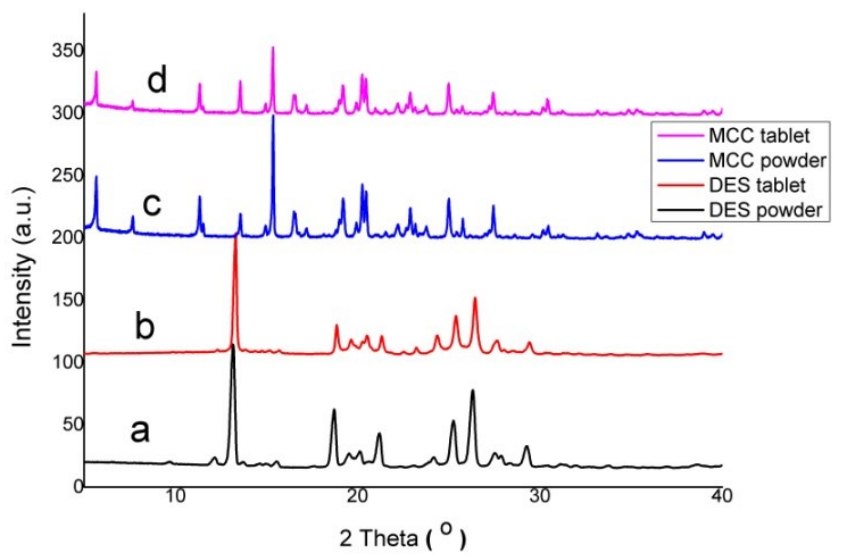

Figure 2. The PXRD patterns: a. Desloratadine (DES) powder; b. DES tablet; c. Multicomponent crystal (MCC) powder and d. MCC tablet.

Crystallinity is defined as the degree of structural order in solid, shown in fraction or percentage as a measurement of how likely atoms or molecules are arranged in a crystal pattern. PXRD is commonly used as 
a standard method to measure crystallinity $[13,17]$. The reduction of the degree of crystallinity was lower in MCC compared to DES. However, the overall changes of crystallinity were relatively slight after compression where pressure during compression did not cause amorphization of those two crystals. This result was in accordance with a study regarding microcrystalline cellulose [18], aspirin, and nicotinic acid [19], which showed a decrease in the degree of crystallinity because of the effect of compression pressure. During compression, solid particle rearranges followed by deformation, fracture and fragmented to compact mass which affects by molecular arrangement in crystal lattice and interaction intermolecular. Reducing of intensity diffractogram showed a crystal defect. Tendency of a decrease in the degree of crystallinity was assigned by boarding of peak and reducing its intensity. This result probably was affected by formation of amorphous in which the degree of crystallinity reduced after compression pressure.

Table 1: Degree of crystallinity for desloratadine (DES) and multicomponent crystal (MCC)

\begin{tabular}{ccc}
\hline & \multicolumn{2}{c}{ Degree of crystallinity $(\%)$} \\
\cline { 2 - 3 } & Powder & Tablet \\
\hline DES & 86.5 & 77.2 \\
MCC & 77.1 & 70.7 \\
\hline
\end{tabular}

\subsubsection{Differential scanning calorimetry (DSC)}

DSC methods measured crystallinity from fusion heat which quantified energy related to molecular interactions in the crystal. However, the same dislocation molecule still needs energy to break the interaction of remaining lattices when it melts, thus contributing to crystallinity which is measured using the DSC method [13,17]. Thermal analysis results from the effect of compression treatment on DES and MCC are presented in Figure 3. DES powder showed an endothermic peak at $157.0^{\circ} \mathrm{C}$, which requires heat of 172.2 $\mathrm{kJ} / \mathrm{g}$ and DES tablet showed an endothermic peak at $160.3{ }^{\circ} \mathrm{C}$, which requires heat of $127.8 \mathrm{~kJ} / \mathrm{g}$. Meanwhile, MCC powder has an endothermic peak at $175.6^{\circ} \mathrm{C}$, which requires heat of $169.7 \mathrm{~kJ} / \mathrm{g}$ and the MCC tablet has an endothermic peak at $178.2^{\circ} \mathrm{C}$, which requires heat of $135.1 \mathrm{~kJ} / \mathrm{g}$.

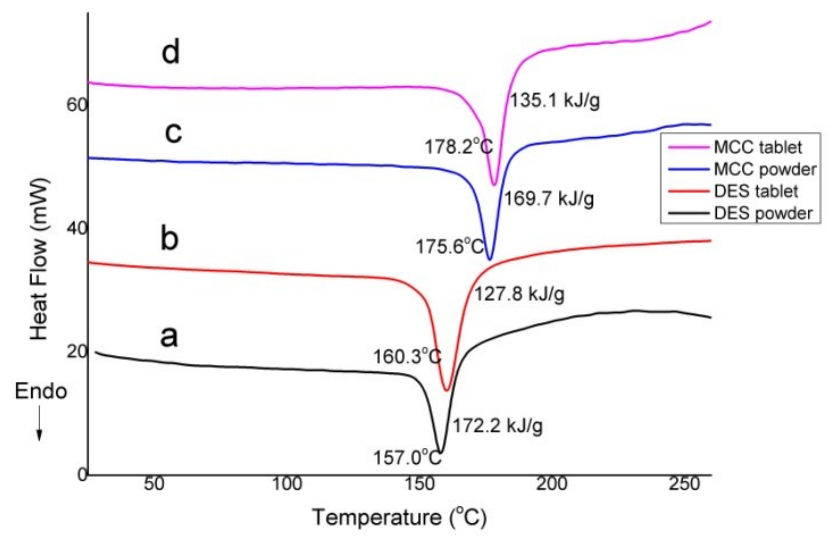

Figure 3. Thermograms of a. desloratadine (DES) powder; b. DES tablet; c. multicomponent crystal (MCC) powder; d. MCC tablet.

DSC is a characterization method based on thermodynamic properties that occur in the samples by observing heat energy produced, which can be observed from the endothermic and exothermic peaks in the DSC thermogram. The decrease of this enthalpy indicated a decrease in crystallinity caused by the tableting compression process. Therefore, heat energy required to melt DES and MCC was lower in tablet than in powder. The decrease of crystallinity affected thermal behavior, where heat energy of DES and MCC decreased after the compression process.

\subsubsection{Fourier transform infrared (FTIR) spectroscopy}

A common method used to characterize drugs is infrared spectra. Infrared spectroscopy (FTIR) analysis is conducted to identify a functional group in compounds. FTIR are sensitive to component 
structure and conformation and can be used to compare structures of components in different solids. Characterization of infrared spectroscopy from DES and MCC powder and tablet is presented in Figure 4. There were no changes observed between functional groups of DES and MCC powder and tablet, which indicated that there were no changes to the crystal substance and chemical structures after the compression process. Because of its sensitivity, this instrument is useful for characterizing pharmaceutical crystals. FTIR spectra after compression show broad peaks when experiencing changes in amorphous form with decreasing degree of crystallinity [20-21], but this study did not show changes in the peak, which showed a low decrease of crystallinity that can only be observed by PXRD and DSC analysis. Principally, absorption of FTIR band before and after compression showed similar features.

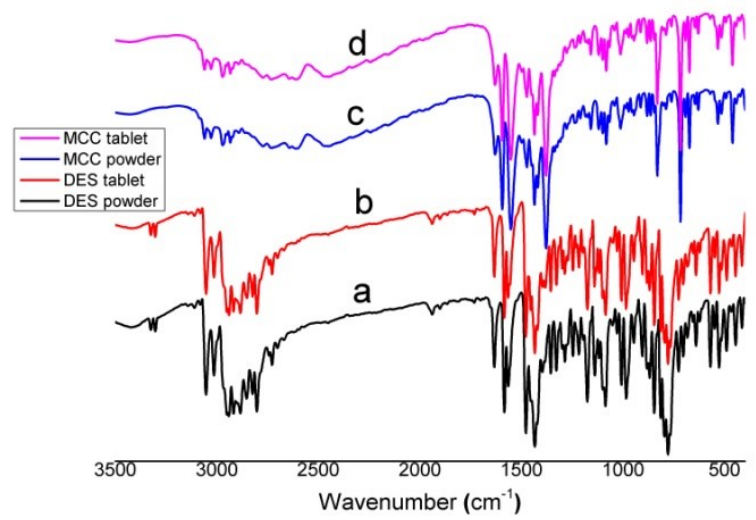

Figure 4. FTIR spectra of a. desloratadine (DES) powder; b. DES tablet; c. multicomponent crystal (MCC) powder; d. MCC tablet.

\subsubsection{Scanning electron microscopy (SEM)}

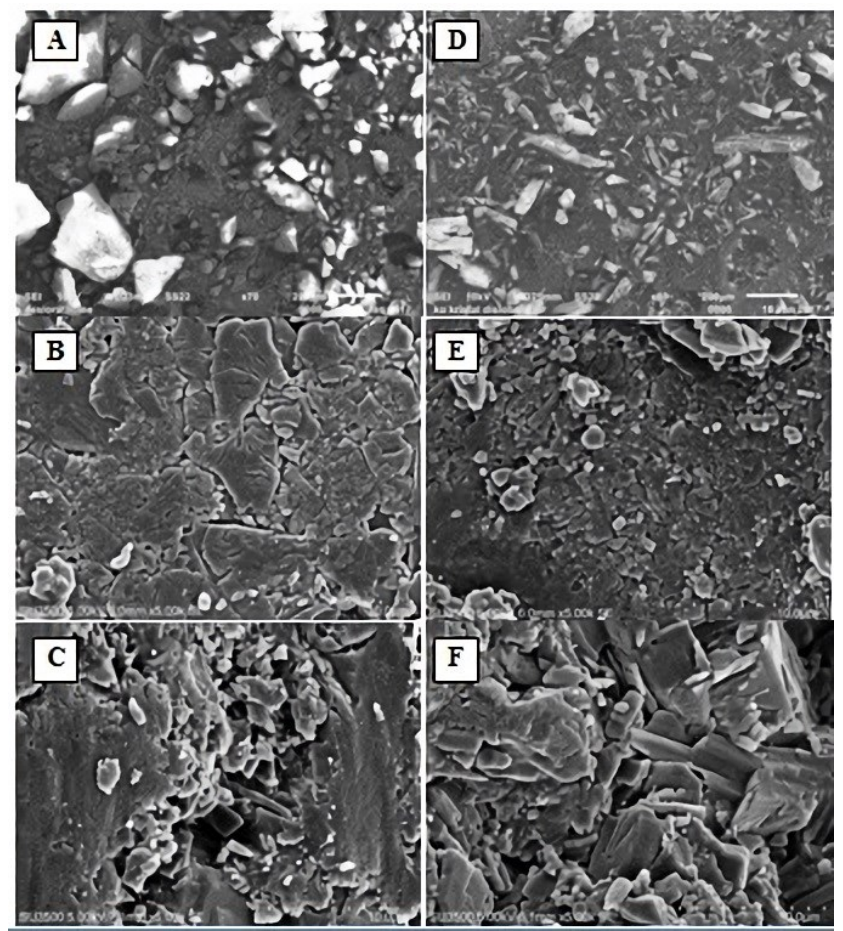

Figure 5. SEM photographs of A.desloratadine (DES) powder; B.surface of DES tablet; C.fragment of DES tablet; D.multicomponent crystal (MCC) powder; E.surface of MCC tablet; and F.fragment of MCC tablet.

That morphology of drug components can be analyzed using SEM [22-23]. SEM is an analysis technique used to observe the changes in particle morphology caused by compression pressure, including 
sintering phenomena. Sintering causes an increase in particle size, adhesion occurs between nearby particles. SEM observation was performed on the surface and fragment parts of the tablet, which are presented in Figure 5. In 5000x magnification, the attachment between DES particles was clearly observed. Fragment parts of the DES tablet showed sintering, which is the loss of surface margin because of particles merging, where primary particles are gone with compression force and showed slight fragments from the sintering. The DES tablet surface showed a sintering effect on the size of particles and morphology. Meanwhile, fragment parts of MCC tablet showed particles that did not attach to themselves while the surface parts of the MCC tablet showed small particles with no evidence of merging to bigger particles. DES showed a weak sintering effect because particle merging still occurred, which increases the size, but has not yet reached the closing of most pores.

\subsection{In vitro dissolution studies}

Observation of dissolution profile was performed on DES and MCC tablets in a dissolution medium of $0.1 \mathrm{~N} \mathrm{HCl}$ and water. Results of dissolution test of DES and MCC tablet are presented in Figure 6. The curve showed the percentage of dissolution tablet was higher in MCC than DES for 1 hour on both dissolution medium. The effect of compression in this study was compared with the dissolution of DES and MCC powder thus produced dissolution efficiency (DE) value is presented in Table 2. Effect of compression reduces the DE value from MCC and DES after being compressed to tablet form compared with before compression for all dissolution mediums. However, the decrease varied from the lowest at $2.06 \%$ DES in $\mathrm{HCl}$ medium, 2.36\% DES in a water medium, 2.38\% $\mathrm{MCC}$ in $\mathrm{HCl}$ medium, and the highest at $7.99 \% \mathrm{MCC}$ in a water medium.

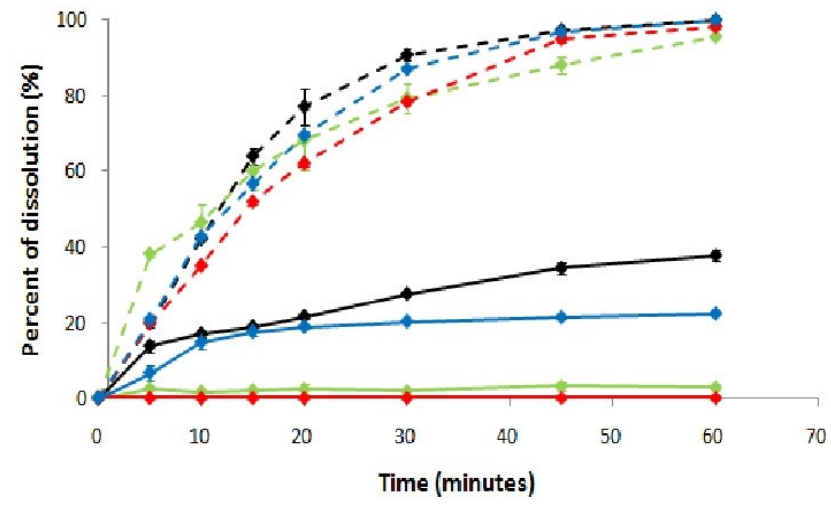

Figure 6. Dissolution profiles of desloratadine powder (green), desloratadine tablet (red), multicomponent crystal powder (black) and multicomponent crystal tablet (blue) in water (solid lines) and $0.1 \mathrm{~N} \mathrm{HCl}$ (dashed lines) dissolution media.

The dissolution decrease occurred in tablets because during compression to form a tablet, dissolution medium may penetrate tablet pores, which can cause tablet breakage to form powder before dissolving. The mechanism of dissolution of DES and MCC tablet takes a longer time because the tablet is a combination of interparticle attachments, which take a longer time to reform to powder. Therefore, the decrease of dissolution in this study was still a reasonable amount not because of sintering phenomena (the loss of tablet surface margin) [24]. Meanwhile, the factor of degree of crystallinity decrease, which increases dissolution or the other way around [25] did not occur here, but what happened was a degree of crystallinity decrease and a decrease in dissolution. Therefore, the crystallinity factor has no effect on dissolution.

Table 2: Dissolution efficiency of desloratadine (DES) and multicomponent crystal (MCC)

\begin{tabular}{lcc}
\hline Dissolution Medium & \multicolumn{2}{c}{ Dissolution Efficiency (\%) } \\
\cline { 2 - 3 } & Powder & Tablet \\
\hline DES in water & $2.48 \pm 0.56$ & $0.12 \pm 0.03$ \\
MCC in water & $25.86 \pm 0.77$ & $17.87 \pm 0.67$ \\
DES in $0.1 \mathrm{~N} \mathrm{HCl}$ & $70.96 \pm 2.60$ & $68.90 \pm 0.21$ \\
MCC in $0.1 \mathrm{~N} \mathrm{HCl}$ & $75.78 \pm 1.27$ & $73.40 \pm 0.14$ \\
\hline
\end{tabular}




\section{CONCLUSION}

The results of this study regarding the effect of tablet compression on physicochemical properties and dissolution of DES and MCC salt showed that there was a decrease of crystallinity before and after undergoing the tablet compression process. Compression to tablet form decreases the enthalpy of DES and MCC. The tableting process caused interparticle merging, which occurred with DES but did not occur with MCC. The dissolution only showed small decreasing changes after being compressed. Confirmation of those physicochemical characterizations showed that there was an effect of compression, but it showed no effect on dissolution.

\section{MATERIALS AND METHODS}

\subsection{Materials}

Desloratadine (pharmaceutical grade) was purchased from Xi' An Wango Biopharm Co., Ltd., (Shaanxi, China). Benzoic acid (analytical grade) and hydrochloric acid (analytical grade) were obtained from Merck (Darmstadt,Germany). Methanol (analytical grade) was obtained from J.T. Baker, Inc (NJ, USA).

\subsection{Multicomponent crystal (MCC) preparation with solvent evaporation}

An equimolar mixture of $1 \mathrm{mmol}$ DES and BA was dissolved in methanol at $35^{\circ} \mathrm{C}$. Formation of MCC salt was carried out using the rapid solvent evaporation method using a Buchi Rotavapor R-215 (Flawil, Switzerland) at $50{ }^{\circ} \mathrm{C}$, continued with Buchi Heating Bath B-491 (Flawil, Switzerland) and vacuumed at 208 mbar pressure using a Buchi vacuum controller V-850. The solid material obtained was collected and made into a tablet.

\subsection{Formation of a tablet}

DES tablet formation was done by accurately weighing powder to $500.0 \pm 2 \mathrm{mg}$, and MCC was equivalently scaled to $696.4 \pm 2 \mathrm{mg}$ and then placed into a $13 \mathrm{~mm}$ diameter tableting die and compressed in $150 \mathrm{MPa}$ pressure for DES and $25 \mathrm{MPa}$ for MCC to produce the same tensile strength which is $1.36 \mathrm{MPa}$ using hydraulic press machine (Perkin Elmer, MA, USA) [10]. Performed on the same tensile strength 1.36 $\mathrm{MPa}$, because it can to compensate the weighted variables that will be dimensions so that the variables can be controlled and the evaluation results can be valid. Before use, each die, upper, and lower punch was lubricated with a thin layer of magnesium stearate powder using a brush.

\subsection{Physicochemical Properties Evaluation}

\subsubsection{Powder X-Ray diffraction (PXRD)}

A sample was placed on the X-ray diffractometer stage. The PXRD pattern was obtained from characterization using Bruker D8 Advance X-Ray Diffractometer (WI, USA) using Cu-ka radiation $(\lambda=$ $1.5406 \AA$ ), $40 \mathrm{kV}$ voltage and $35 \mathrm{~mA}$ current. The scan was performed at a rate of $2^{\circ} /$ minute with a diffraction angle of $2 \theta$ from 5 to $60^{\circ}$ and intensity interval of $0.02^{\circ}$. Subsequently, formed diffraction peaks were compared. Crystallinity analysis was performed by plotting $X, Y$ which are $2 \theta$ vs intensity, respectively, using OriginPro 9.0 software. Smoothing was conducted by taking 15 points with adjacent averaging methods. Afterwards, a straight line was substracted to make $Y=0$. A change in crystal pattern was compared from crystalline component peak area ratio with total peak area which is a measure of the degree of crystallinity (\%) as measured using OriginPro 9.0 software [6].

\subsubsection{Differential Scanning Calorimetry (DSC)}

Characterization of DSC was performed using LINSEIS STA PT-1600 (NJ, USA) which was calibrated for cell constant and temperature using indium. The following parameters were used: temperature $25-500{ }^{\circ} \mathrm{C}$ under a nitrogen purge $100 \mathrm{~mL} / \mathrm{min}$, heating rate of $10^{\circ} / \mathrm{min}, \mathrm{Al}_{2} \mathrm{O}_{3}$ crucible; and 2-5 mg samples were placed into the pan.

\subsubsection{Fourier transform infrared (FTIR) spectroscopy}

IR spectrum sample identification was performed using FTIR IR Prestige-21 Shimadzu (Kyoto, Japan) analyzed in the wavenumber range 4000-400 $\mathrm{cm}^{-1}$ with $2 \mathrm{~cm}^{-1}$ resolution. Afterwards, the obtained spectrum peaks were compared. Intensity and shifting of vibrational peaks was observed. 


\subsubsection{Scanning electron microscopy (SEM)}

Each tablet surface and fragment were placed into a sample holder and layered with $2.5 \mathrm{~nm}$ gold using HITACHI MC1000 ION SPUTTER (Tokyo, Japan). Samples layered with gold were subsequently placed in the specimen chamber of a HITACHI SU3500 scanning electron microscope (Tokyo, Japan) and observed on host computer for proper image and magnification. Voltage and current were set at $5 \mathrm{kV}$ and 10 $\mathrm{mA}$. The photomicrograph of surface morphology and cross-section of tablets DES and MCC were compared.

\subsection{In Vitro dissolution studies}

Dissolution experiments were conducted with DES and MCC tablets which were placed into a dissolution vessel with $0.1 \mathrm{~N} \mathrm{HCl}$ and water medium $900 \mathrm{~mL}$, with temperature of $37 \pm 0.5{ }^{\circ} \mathrm{C}$, stirring rate at $50 \mathrm{rpm}$, using Hanson Virtual Instruments SR8Plus Dissolution test station (Hanson Research, CA, USA) according to USP apparatus II (paddle method). $5 \mathrm{~mL}$ samples were taken at 5, 10, 15, 20,30, 45, and 60 minutes time intervals, then changed with $5 \mathrm{~mL}$ of new dissolution medium. Aliquots were filtered using $0.45 \mu \mathrm{m}$ filter membrane and its content was analyzed using Beckman Coulter DU ${ }^{\circledR 720}$ General Purpose Spectrophotometer (CA, USA) at a validated wavelength of $290 \mathrm{~nm}$. Resulting data were analyzed to obtain dissolution efficiency (DE).

Acknowledgements: This paper is a partially of Ahmad Ainurofiq's Ph.D. thesis submitted to the School of Pharmacy, Institut Teknologi Bandung. It was financially supported by "Hibah Program Penelitian, Pengabdian kepada Masyarakat, dan Inovasi (P3MI) Kelompok Keahlian ITB".

Author contributions: Concept - A.A., S.N.S., R.M., D.M.; Design - A.A., S.N.S.; Supervision - R.M., D.M.; Resource S.N.S., R.M., D.M.; Materials - R.M., D.M.; Data Collection and/or Processing - A.A., S.N.S.; Analysis and/or Interpretation - A.A., S.N.S.; Literature Search - R.M., D.M.; Writing - A.A., S.N.S.; Critical Reviews - A.A., S.N.S., R.M., D.M.

Conflict of interest statement: The authors declare no conflict of interest.

\section{REFERENCES}

[1] Mazel V, Delplace C, Busignies V, Faivre V, Tchoreloff P, Yagoubi N. Polymorphic transformation of anhydrous caffeine under compression and grinding: a re-evaluation. Drug Dev Ind Pharm. 2011;37(7):832-840. [CrossRef]

[2] Patel S, Kaushal AM, Bansal AK. Compression physics in the formulation development of tablets. Crit Rev Ther Drug Carrier Syst. 2006;23(1):1-65. [CrossRef]

[3] Brittain HG. Effects of mechanical processing on phase composition. J Pharm Sci. 2002;91(7):1573-1580. [CrossRef]

[4] Morris KR, Griesser UJ, Eckhardt CJ, Stowell JG. Theoretical approaches to physical transformations of active pharmaceutical ingredients during manufacturing processes. Adv Drug Deliv Rev. 2001;48(1):91-114. [CrossRef]

[5] Feng T, Pinal R, Carvajal MT. Process induced disorder in crystalline materials: Differentiating defective crystals from the amorphous form of griseofulvin. J Pharm Sci. 2008;97(8):3207-3221. [CrossRef]

[6] Bhadra S, Khastgir D. Determination of crystal structure of polyaniline and substituted polyanilines through powder X-ray diffraction analysis. Polym Test. 2008;27(7):851-857. [CrossRef]

[7] Anthes JC, Gilchrest H, Richard C, Eckel S, Hesk D, West RE, Williams SM, Greenfeder S, Billah M, Kreutner W, Egan RW. Biochemical characterization of desloratadine, a potent antagonist of the human histamine $\mathrm{H}(1)$ receptor. Eur J Pharmacol. 2002;449(3):229-237. [CrossRef]

[8] Geha RS, Meltzer EO. Desloratadine: A new, nonsedating, oral antihistamine. J Allergy Clin Immunol. 2001;107(4):751-762. [CrossRef]

[9] Agrawal DK. Pharmacology and clinical efficacy of desloratadine as an anti-allergic and anti-inflammatory drug. Expert Opin Investig Drugs. 2001;10(3):547-560. [CrossRef]

[10] Ainurofiq A, Mauludin R, Mudhakir D, Umeda D, Soewandhi SN, Putra OD, Yonemochi E. Improving mechanical properties of desloratadine via multicomponent crystal formation. Eur J Pharm Sci. 2018;111:65-72. [CrossRef]

[11] Ahmed H, Shimpi MR, Velaga SP. Relationship between mechanical properties and crystal structure in cocrystals and salt of paracetamol. Drug Dev Ind Pharm. 2017;43(1):89-97. [CrossRef] 
[12] Chang S-Y, Sun CC. Superior Plasticity and Tabletability of Theophylline Monohydrate. Mol Pharm. 2017;14(6):2047-2055. [CrossRef]

[13] ter Laak AM, Tsai RS, Donné-Op den Kelder GM, Carrupt P-A, Testa B, Timmerman H. Lipophilicity and hydrogen-bonding capacity of H1-antihistaminic agents in relation to their central sedative side-effects. Eur J Pharm Sci. 1994;2(5):373-384. [CrossRef]

[14] Ainurofiq A, Mauludin R, Mudhakir D, Soewandhi SN. Synthesis, characterization, and stability study of desloratadine multicomponent crystal formation. Res Pharm Sci. 2018;13(2):93-102. [CrossRef]

[15] Bernardi LS, Oliveira PR, Murakami FS, Silva M a. S, Borgmann SHM, Cardoso SG. Characterization of venlafaxine hydrochloride and compatibility studies with pharmaceutical excipients. J Therm Anal Calorim. 2009;97(2):729-733. [CrossRef]

[16] Kaneniwa N, Imagawa K, Otsuka M. Effect of Tabletting on the Degree of Crystallinity and on the Dehydration and Decomposition Points of Cephalexin Crystalline Powder. Chem Pharm Bull (Tokyo). 1985;33(2):802-809. [CrossRef]

[17] Lima MFS, Vasconcellos MAZ, Samios D. Crystallinity changes in plastically deformed isotactic polypropylene evaluated by x-ray diffraction and differential scanning calorimetry methods. J Polym Sci Part B Polym Phys. 2002;40(9):896-903. [CrossRef]

[18] Ek R, Wormald P, Iversen T, Nyström C. Crystallinity index of microcrystalline cellulose particles compressed into tablets. Int J Pharm. 1995;125(2):257-264. [CrossRef]

[19] Fukuoka E, Makita M, Yamamura S. Preferred Orientation of Crystallites in Tablets. III. Variations of Crystallinity and Crystallite Size of Pharmaceuticals with Compression. Chem Pharm Bull (Tokyo). 1993;41(3):595-598. [CrossRef]

[20] Brittain HG. Spectral methods for the characterization of polymorphs and solvates. J Pharm Sci. 1997;86(4):405-412. [CrossRef]

[21] Tajber L, Corrigan OI, Healy AM. Physicochemical evaluation of PVP-thiazide diuretic interactions in co-spraydried composites - analysis of glass transition composition relationships. Eur J Pharm Sci. 2005;24(5):553-563. [CrossRef]

[22] Tedesco E, Giron D, Pfeffer S. Crystal structure elucidation and morphology study of pharmaceuticals in development. CrystEngComm. 2002;4(67):393-400. [CrossRef]

[23] Wang QB, Wang QG, Wan CX. Effect of sintering time on the microstructure and properties of inorganic polyphosphate bioceramics. Sci Sinter. 2010;42(3):337-343. [CrossRef]

[24] Riippi M, Yliruusi J, Niskanen T, Kiesvaara J. Dependence between dissolution rate and porosity of compressed erythromycin acistrate tablets. Eur J Pharm Biopharm. 1998;46(2):169-175. [CrossRef]

[25] Suzuki T, Nakagami H. Effect of crystallinity of microcrystalline cellulose on the compactability and dissolution of tablets. Eur J Pharm Biopharm. 1999;47(3):225-230. [CrossRef] 